An English Draft of Dr. Yong Wang

\title{
Relation of Web Service Orchestration, Abstract Process, Web Service and Choreography
}

\author{
Yong Wang \\ College of Computer Science and Technology, \\ Faculty of Information Technology, \\ Beijing University of Technology, Beijing, China
}

\begin{abstract}
We refine the relation of Web service orchestration, abstract process, Web service, and Web service choreography in Web service composition, under the situation of cross-organizational corporation. We also introduce the formal verification process of this relation through an example.
\end{abstract}

Keywords: Web Service; Web Service Orchestration; Web Service Choreography; Abstraction Process

\section{Introduction}

Web service composition is a core mechanism in Web service research domain, it can compose component Web services (WSs) into bigger granularity of WSs. There are two aspects of Web service composition: one is Web service orchestration (WSO) which usually has an abstract process (AB), the other is Web service choreography (WSC). In a framework of formal model of Web service composition [1, we designed an architecture of Web service composition under the situation of business to business (B2B) corporation. In this paper, we refine this architecture to clarify the relation of WSO, AB, WS, and WSC, and introduce the formal verification process of this relation by use of truly concurrent process algebra APTC [2] through an example.

This paper is organized as follows. We do not introduce the preliminaries on truly concurrent process algebra APTC and Web service composition, please refer to [2] and 1] for details. In section 2, we refine the relation of WSO, AB, WS, and WSC. We introduce the formal verification process of the relation by APTC through an example in section 3 . Finally, in section 4, we conclude this paper.

Correspondence and offprint requests to: Yong Wang, Pingleyuan 100, Chaoyang District, Beijing, China. e-mail: wangy@bjut.edu.cn 


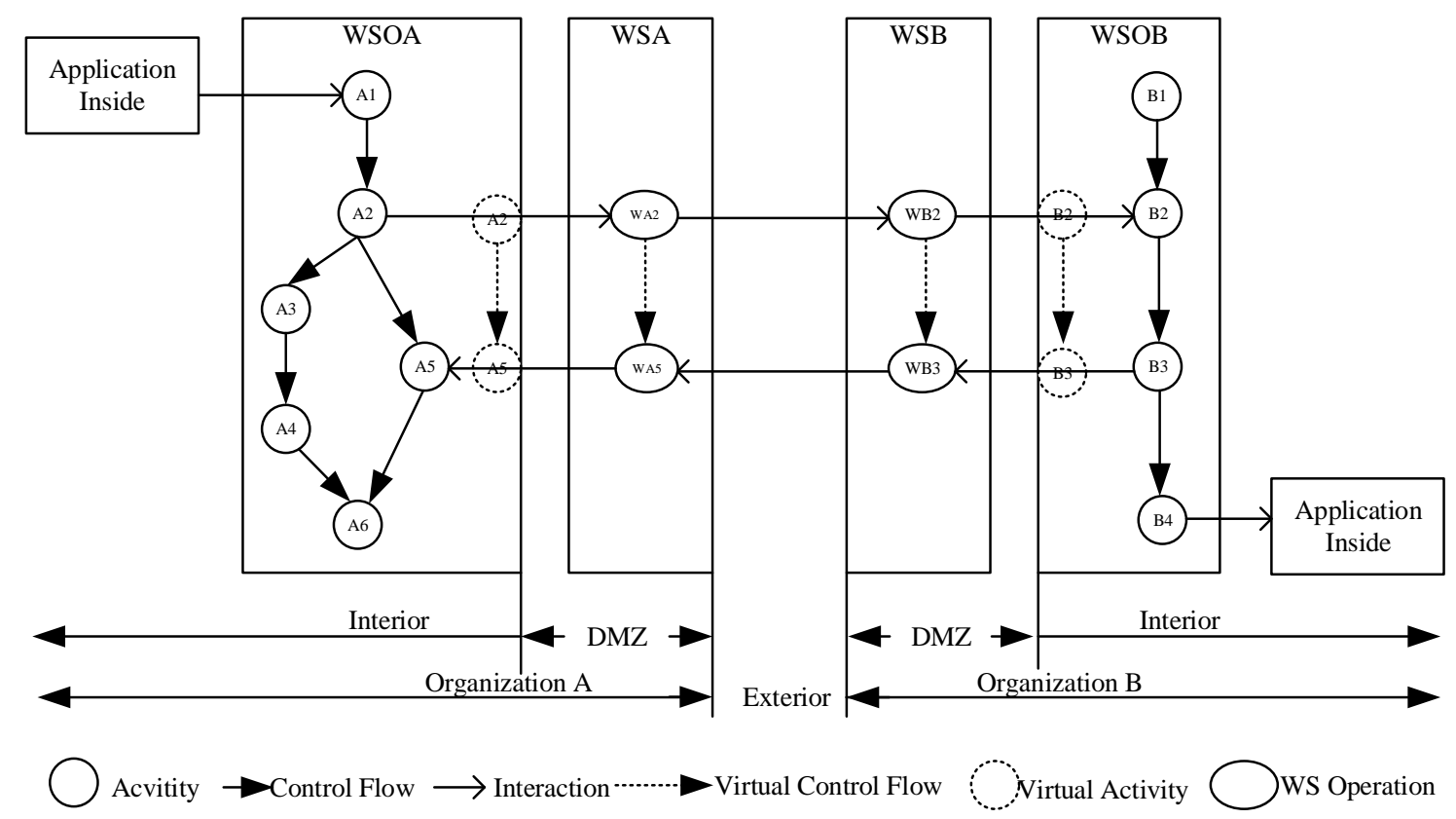

Fig. 1. Relation of WSO, AB, WS and WSC.

\section{Relation of WSO, AB, WS and WSC}

The relation among WSO, AB, WS and WSC are illustrated in Fig. 1. It was firstly occurred in a formal model of Web service composition [1, and we detail this relation here.

1. WSO: A WSO is an orchestration of workflow activities, including internal activities, activities interacting with internal applications, activities interacting with WSs outside via its interface WS. WSO locates in the interior of organization and usually is executed with in WSO engine;

2. AB: An abstract process is an abstraction of a WSO by eliminating the inner activities and activities interacting with inner applications, it is only a description of the interface of a WSO with virtual control flow and data flow, and it can not usually be executed. An AB corresponds to its interface WS exactly with each virtual activity corresponding to a WS operation in its interface WS;

3. WS: A WS is the real interface of a WSO interacting with other WSOs or WSs outside, and it is located in the DMZ and may be equipped within a Web (Service) server;

4. WSC: A WSC is the specification of interactions between WSs, it may have an entity or just act as a contract of involved WSs.

\section{Verification of the Relation}

The above relation can be formally verified by truly concurrent process algebra APTC [2]. The verification process is following.

1. Giving the APTC descriptions of WSOs, WSs;

2. By abstracting the internal activities, activities interacting with internal applications, the ABs can be obtained; 
3. Putting WSOs, WSs in parallel, treating interactions between WSO and WS, WS and WS, WS and WSO as internal actions, and by use of recursion, we can verify the whole system if is correct.

We take the example illustrated in Fig. 1, and verify its correctness.

The APTC description of WSOA is following.

$W S O A=\sum_{d \in \Delta} A 1(d) \cdot W S O A 1$

$W S O A 1=A 2 \cdot W S O A 2$

$W S O A 2=((A 3 \cdot A 4) \| A 5) \cdot W S O A 3$

$W S O A 3=A 6 \cdot W S O A$

By defining $I=\{A 1(d), A 3, A 4, A 6\}$, we can get $A B A=\tau_{I}(W S O A)=A 2 \cdot A 5$.

The APTC description of WSA is as follows. Note that, to make the interacting action in the same causal depth, we use the shadow constant.

$W S A=S_{A 1} \cdot W S A 1$

$W S A 1=W A 2 \cdot W S A 2$

$W S A 2=W A 5 \cdot W S A 3$

$W S A 3=S_{A 6} W S A$

The APTC description of WSOB is following.

$W S O B=B 1 \cdot W S O B 1$

$W S O B 1=B 2 \cdot W S O B 2$

$W S O B 2=B 3 \cdot W S O B 3$

$W S O B 3=\sum_{d^{\prime} \in \Delta} B 4\left(d^{\prime}\right) \cdot W S O B$

By defining $I=\{B 1, B 4\}$, we can get $A B B=B 2 \cdot B 4$.

The APTC description of WSB is as follows. Note that we also use the shadow constant for the same reason.

$W S B=\mathrm{S}_{B 1} \cdot W S B 1$

$W S B 1=W B 2 \cdot W S B 2$

$W S B 2=W B 3 \cdot W S B 3$

$W S B 3=\mathrm{SS}_{B 4} \cdot W S B$

We define the following communication function, and the communications between any other two atomic actions will cause deadlock.

$\gamma(A 2, W A 2) \triangleq c(A 2, W A 2)$

$\gamma(A 5, W A 5) \triangleq c(A 5, W A 5)$

$\gamma(B 2, W B 2) \triangleq c(B 2, W B 2)$

$\gamma(B 3, W B 3) \triangleq c(B 3, W B 3)$

$\gamma(W A 2, W B 2) \triangleq c(W A 2, W B 2)$

$\gamma(W A 5, W B 3) \triangleq c(W A 5, W B 3)$

Then, we define $I=\left\{A 2, A 3, A 4, A 5, A 6\right.$, $\mathrm{S}_{A 6}, W A 2, W A 5, W B 2, W B 3, B 1, \mathrm{~S}_{B 1}, B 2, B 3$, $c(A 2, W A 2), c(A 5, W A 5), c(B 2, W B 2), c(B 3, W B 3), c(W A 2, W B 2), c(W A 5, W B 3)\}$

We can get the system $\tau_{I}\left(\partial_{H}(\Theta(W S O A \searrow W S A \searrow W S B \searrow W S O B))\right)$, and the following result.

Theorem 3.1. The system $\tau_{I}\left(\partial_{H}(\Theta(W S O A \searrow W S A \searrow W S B \searrow W S O B))\right)$ has desired external behaviors.

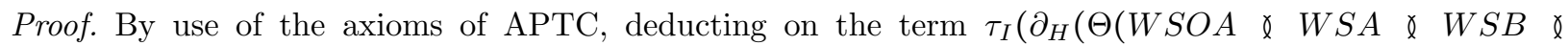
$W S O B))$ ), we can get

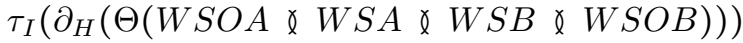

$=\sum_{d \in \Delta} \sum_{d^{\prime} \in \Delta} A 1(d) \cdot B 4\left(d^{\prime}\right) \cdot \tau_{I}\left(\partial_{H}(\Theta(W S O A \succsim W S A \succsim W S B \succsim W S O B))\right)$

\section{Conclusions}

We give the refined relation among WSO, AB, WS and WSC based on a framework of Web service composition [1, and introduce the formal verification process of the relation. Through an example illustrated in Fig. 1. we detail the verification process. 


\section{References}

[1] Y. Wang. Formal Model of Web Service Composition: An Actor-Based Approach to Unifying Orchestration and Choreography. 2013. arXiv:1312.0677

[2] Y. Wang. Algebraic laws for true concurrency. 2016. arXiv: 1611.09035. 